\title{
The global electroweak fit and constraints on new physics with Gfitter
}

Dörthe Ludwig* for the Gfitter group

DESY Hamburg, Unversity of Hamburg

E-mail: doerthe.ludwig@desy.de

The thourough investigation of radiative corrections allows to gain information on physics processes at higher energy scales than those directly accessible by current experiments. As a consequence, using electroweak precision measurements in conjunction with state-of-the-art SM predictions e.g. allows the estimation of a preferred mass range for the SM Higgs boson mass. Physics beyond the Standard Model can modify the relations between electroweak observables and their theoretical predictions. Such effects can be parametrized in terms of effective, so-called oblique parameters. A global fit of the electroweak SM, as performed with the Gfitter package [1], allows the determination of the oblique parameters and to probe physics models and to set constraints on their free parameters. In this paper we present updated results of the global electroweak SM fit taking into account the latest experimental precision measurements and the results of direct Higgs searches from LEP and Tevatron. Through the formalism of oblique parameters we obtain constraints on BSM models with universal and warped extra dimensions. In constrast, taking into account heavy flavor observables, $(\mathrm{g}-2)_{\mu}$, and dark matter predictions allows to constrain the parameter space of the minimal supergravity model (mSugra).

35th International Conference of High Energy Physics - ICHEP2010,

July 22-28, 2010

Paris France

* Speaker. 


\section{The global electroweak fit}

In the global electroweak fit predictions for precision observables are compared with the most recent measurements done by LEP, SLC, and Tevatron. A detailed list of all data used in the fit can be found in [1]. The free floating parameters are $M_{Z}, M_{H}, m_{t}, m_{b}, m_{c}, \Delta \alpha_{\mathrm{had}}^{(5)}$, and $\alpha_{S}\left(M_{Z}^{2}\right)$ where the latest average for the $m_{t}$ as well as the newly obtained exclusion limits for $M_{H}$ [2] have been used.

The fit converges at a $\chi^{2}$ minimum of $16.4(17.8)$ excluding (including) the direct Higgs searches. The respective p-values based on toy Monte Carlo experiments are $0.23(0.22)$ where no individual pull value exceeds $3 \sigma$. One of the most important results of the electroweak fit is the estimation of Higgs mass. The $\chi_{\min }^{2}$ is found at $M_{H}=84.2_{-23.3}^{+30.3} \mathrm{GeV}\left(M_{H}=120.6_{-5.2}^{+17.0} \mathrm{GeV}\right)$ with a $2 \sigma$ interval of $[40.3,159.2] \mathrm{GeV}([114.4,154.9] \mathrm{GeV})$. Figure 1 (left) shows the $\Delta \chi^{2}$ profile as a function of $M_{H}$ for the fit including the direct Higgs searches. The increase of $\Delta \chi^{2}$ at the $95 \% \mathrm{CL}$ exclusion limits from LEP and Tevatron (shaded areas) is clearly visible.

\section{The oblique parameters}

The main assumption that led to the introduction of the oblique paramters [3] is that highscale BSM physics appears only through vacuum polarisation corrections. The electroweak fit is sensitive to BSM physics through these oblique corrections which can be described through the $S T U$ parametrization: $O_{\text {meas }}=O_{S M ; r e f}\left(M_{H} ; m_{t}\right)+c_{S} S+c_{T} T+c_{U} U$. The $S T U$ parameters measure deviations from electroweak radiative correction that are expected in the reference SM determined by the chosen $m_{t}$ and $M_{H}$.

In our analysis the $\mathrm{SM}$ reference point is chosen to be at $M_{H}=120 \mathrm{GeV}$ and $\mathrm{m}_{\mathrm{t}}=173.3 \mathrm{GeV}$. $S, T$, and $U$ are derived from a fit to electroweak observables and are compatible with 0 as illustrated in Fig. 1 (right) showing the 68\%, 95\%, and 99\% CL level allowed regions in the $S$ - $T$-plane for $U=0$. The grey area shows the SM prediction highlighting the logarithmical dependence of $S$ and $T$ on $M_{H}$. Small values of $M_{H}$ are compatible with data.
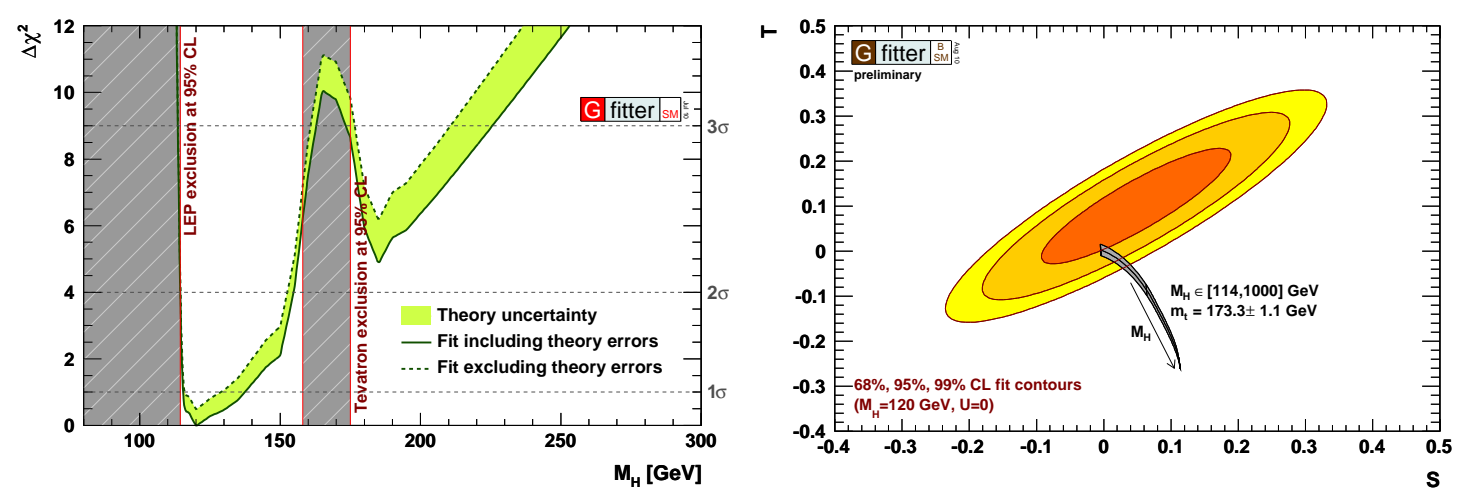

Figure 1: Left: $\Delta \chi^{2}$ profile as a function of $M_{H}$ for the electroweak fit including results of direct Higgs searches at LEP and Tevatron (shaded areas). Right: Fit result of the oblique parameters. Shown are the $68 \%, 95 \%$, and $99 \% \mathrm{CL}$ allowed regions in the $S$ - $T$-plane with $U=0$ for a reference SM with $M_{H}=120$ $\mathrm{GeV}$ and $m_{t}=173.3 \mathrm{GeV}$. The grey area illustrates the SM prediction for various values of $M_{H}$ and $m_{t}$. 

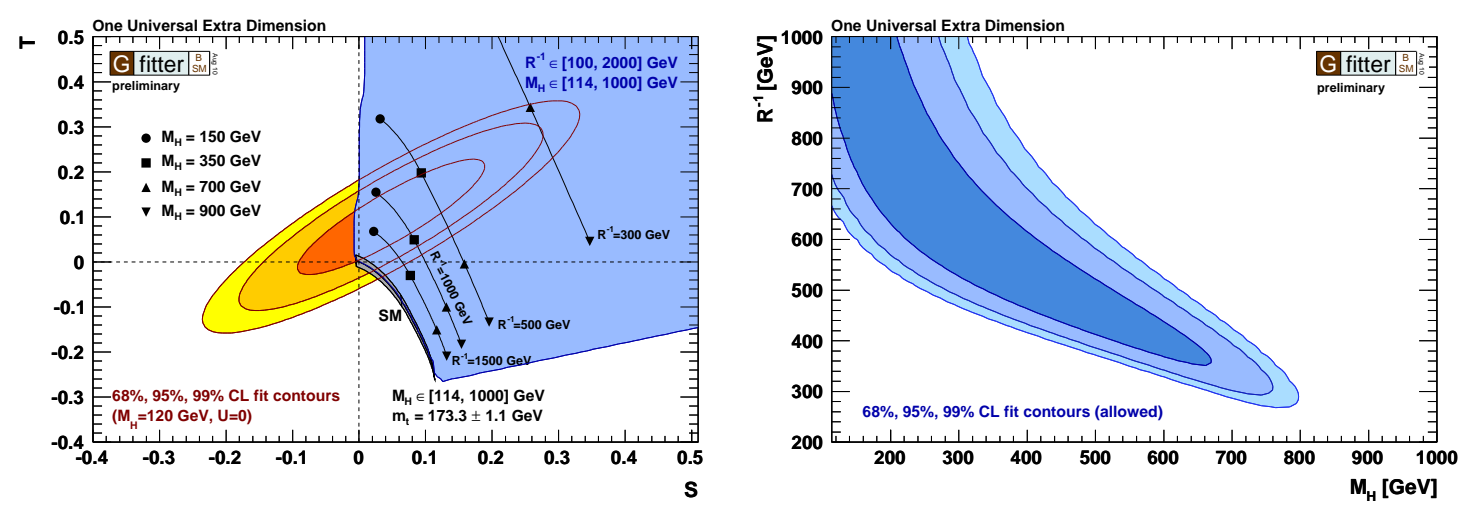

Figure 2: Left: Comparison in the S-T-plane between CL contours from fits to the electroweak precision data and predictions of the UED model. For illustration some benchmark points are depicted. With increasing compactification scale the ST values converge to the SM predictions. Right: Contours of 68\%, $95 \%$ and 99\% CL obtained from scans of fits with fixed variable pairs $R^{-1}$ and $\mathrm{M}_{\mathrm{H}}$.

\section{Extra dimension models}

The universal extra dimension model (UED) [4] allows all SM particles to propagate into the extra dimensions. Their compactification leads to Kaluza-Klein (KK) modes. The free parameters of this model are the number of extra dimensions $d_{\text {UED }}$ which is fixed to one in our analysis and the compactification scale $R^{-1}$ where $R$ is the size of the extra dimension. The $S, T$, and $U$ parameters mainly depend $R^{-1}, M_{H}$, and $m_{t}$. The result is illustrated in Fig. 2 (left). The blue region indicates the $S$ - $T$-parameter space allowed for various parameter sets in the UED model. For large compactification it approaches the SM prediction. Only small $M_{H}$ are allowed. If the compactification becomes smaller the BSM contribution needs to be compensated by larger $M_{H}$. A comparison with electroweak precision data allows the exclusion of $R^{-1}<300 \mathrm{GeV}$ and $M_{H}>800 \mathrm{GeV}$ as can be seen in Fig. 2(right) in which the 68\%, 95\%, and 99\% CL allowed regions are shown.

A different higher-dimensional approach has been proposed by Randall and Sundrum[5]. The geometry of this model is characterized by one warped extra dimension confined by two threebranes, one of them containing the SM fields. The generation of the weak scale from a larger scale is achieved by introducing a warp factor altering the known four-dimensional metric. The inverse of the warp factor $L$ and the Kaluza-Klein scale $M_{\mathrm{KK}}$ are free model parameters. In a slightly extended version of the original minimal model SM gauge bosons and fermions can propagate in the five-dimensional warped bulk region [6]. Figure 3 (left) shows again in blue the allowed region in the $S$ - $T$-plane. For large values of $L$ high $M_{\mathrm{KK}}$ values are preferred by the data. However, the higher $M_{H}$ the lower $M_{\mathrm{KK}}$ values can be compensated.

\section{Minimal Supergravity}

Minimal supergravity is one of the most investigated supersymmetry models by current collider experiments. One highly constraining breaking mechanism suggests that the breaking is mediated by the gravitational interaction. Some of the free parameters of this model are $m_{0(12)}$, the mass of scalar particles (fermions) at the GUT scale, and $\tan \beta$, the ratio of the two Higgs vacuum 

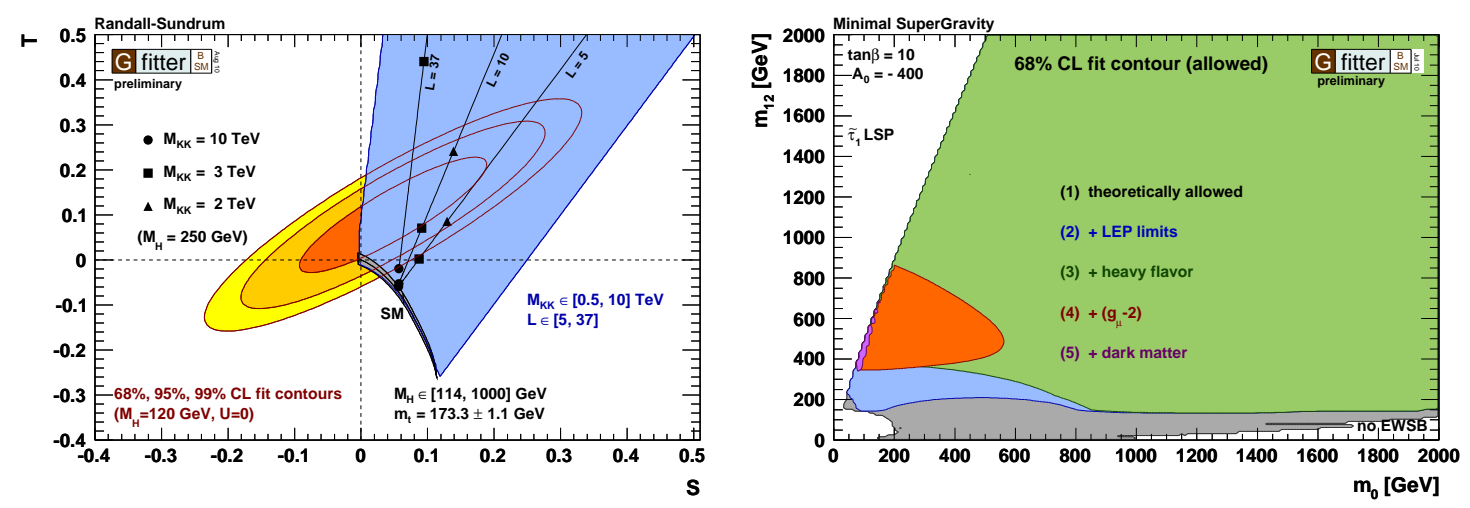

Figure 3: Left: Comparison in the S-T-plane between CL contours from fits to the electroweak precision data and predictions from models with one warped extra dimensions. For illustration several benchmark points are depicted. Right: Contours of $68 \% \mathrm{CL}$ obtained from scans with fixed $m_{0}-m_{12}$ pairs taking into account constraints from (2) LEP (blue), (3)heavy flavor (green), (4) $(g-2)_{\mu}$ (orange), and (5) dark matter (purple).

expectation values. Figure 3 (right) shows the constraints on $m_{0}$ and $m_{12}$. Whereas the impact of the LEP and heavy flavor data do not severely limit these two parameters the $(g-2)_{\mu}$ and dark matter measurements clearly favor small values.

\section{Conclusion}

The global electroweak fit shows an excellent agreement of the SM with data. Including the latest experimental results from Tevatron for $m_{t}, M_{W}$, and $M_{H}$ results in a $\chi_{\min }^{2}$ at $M_{H}=$ $120.6_{-5.2}^{+17.0} \mathrm{GeV}$. However, this result may change if BSM physics is present. For many new physics model contributions from a larger Higgs mass could be compensated and be still compatible with current data. A more detailed list including the Littlest Higgs model, warped extra dimensions with custodial symmetry and a fourth generation model can be found in [1]. As the LHC and Tevatron contribute further to the electroweak precision measurements tighter constraints might be set on many BSM models. Therefore, a continuous development of the electroweak fit and the oblique parameters will be carried out.

\section{References}

[1] H. Flächer et al., Eur. Phys. J. C, 60, 543 (2009), updates and newly obtained results available at www.cern.ch/gfitter.

[2] Tevatron Electroweak Working group, arXiv:1007.3178v1 [hep-ex], 2010; Ben Kilminster, these proceedings.

[3] M. E. Peskin and T. Takeuchi. Phys. Rev. D 46, 381 (1991)

[4] T. Appelquist and H. Yee, Phys. Rev. D 67, 055002 (2003); I. Gogoladze and C. Macesanu, Phys.Rev. D 74, 093012 (2006).

[5] L. Randall and R. Sundrum, Phys. Rev. Lett. 83, 3370 (1999).

[6] S.Casagrande et al., JHEP 10 (2008) 094. 